\title{
Editorial
}

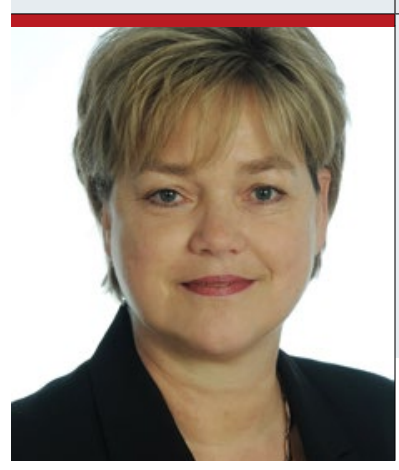

Andrea Tauchert

Leitung Kongressorganisation

andrea.tauchert@springer.com

facebook.com/gesundheitskongresse

\section{Kongress Pflege 2016}

Zusammenkommen ist ein Anfang, zusammenbleiben ist ein Fortschritt und zusammenarbeiten ist ein Erfolg.

Dieses Zitat von Henry Ford bewegt mich gerade in diesen turbulenten Zeiten, in denen es immer mehr darauf ankommt, sich zu vertrauen, aufeinander zu verlassen und gegenseitig wertzuschätzen. Das betrifft uns bei der Organisation des Kongresses Pflege 2016, der am 22. und 23. Januar wieder im Maritim proArte Hotel in Berlin stattfindet, genauso wie Sie, liebe Pflegende, die tagtäglich zusammen mit anderen Berufsgruppen die Patienten versorgen.

\section{Das Team als Klangkörper}

Es ist kein Zufall, dass wir für die Kongresseröffnung einen Dirigenten gewinnen konnten, der uns am Beispiel eines Orchesters deutlich macht, wie wichtig es ist, nicht nur zusammenzukommen, sondern auch gut zusammenzuarbeiten. Sie sind die größte Berufsgruppe im Gesundheitswesen und spielen damit viele Instrumente im großen Orchester der Gesundheitspolitik. Seien Sie sich bewusst, dass jedes Instrument seine Rolle im Ensemble hat und Missstimmungen verursachen würde, wenn es fehlt. Deshalb ist es wichtig, nicht nur die erste Geige wertzuschätzen, sondern auch den Kontrabass und die Triangel.

Wir wollen Ihnen mit dem 21. Kongress Pflege die notwendigen pflegefachlichen Kenntnisse mitgeben und Sie weiter darin bestärken, mit den anderen Beschäftigten im Gesundheitswesen auf Augenhöhe $\mathrm{zu}$ interagieren und sich in das politische Spiel einzumischen. Mit kompetenten Referenten und innovativen Vorträgen haben wir wieder ein Programm von hoher Fachlichkeit und starkem Praxisbezug für alle professionell Pflegenden zusammengestellt, das es in dieser Form nicht noch einmal gibt.

Ich lade Sie herzlich nach Berlin ein, um anlässlich des Kongresses Pflege 2016 zusammenzukommen, und wünsche Ihnen einen guten Start in ein erfolgreiches Jahr 2016.

\section{Auf ein gutes Zusammenspiel!}

Ihre

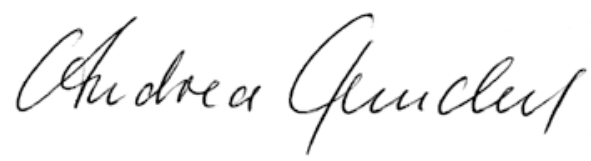

\section{INFO}

\section{HEILBERUFE - die Ausgabe zum Kongress Pflege 2016}

Wenn Referenten zu Autoren werden - auch in diesem Jahr haben wir Themen des Kongresses in der aktuellen Ausgabe von HEILBERUFE aufgriffen. Damit haben auch all diejenigen eine Chance am Kongressgeschehen teilzuhaben, die nicht vor Ort dabei sein können. Mit dem Logo weisen wir auf Vorträge und Inhalte des Kongresses hin. Oder möchten Sie doch noch dabei sein? Dann melden Sie sich rasch an! www.gesundheitskongresse.de

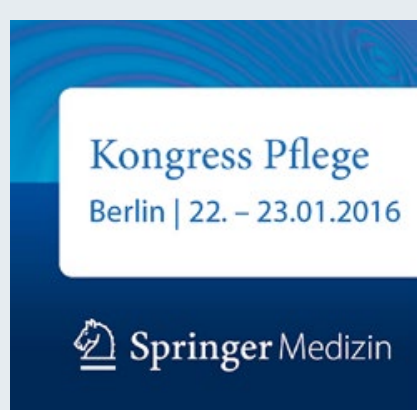

